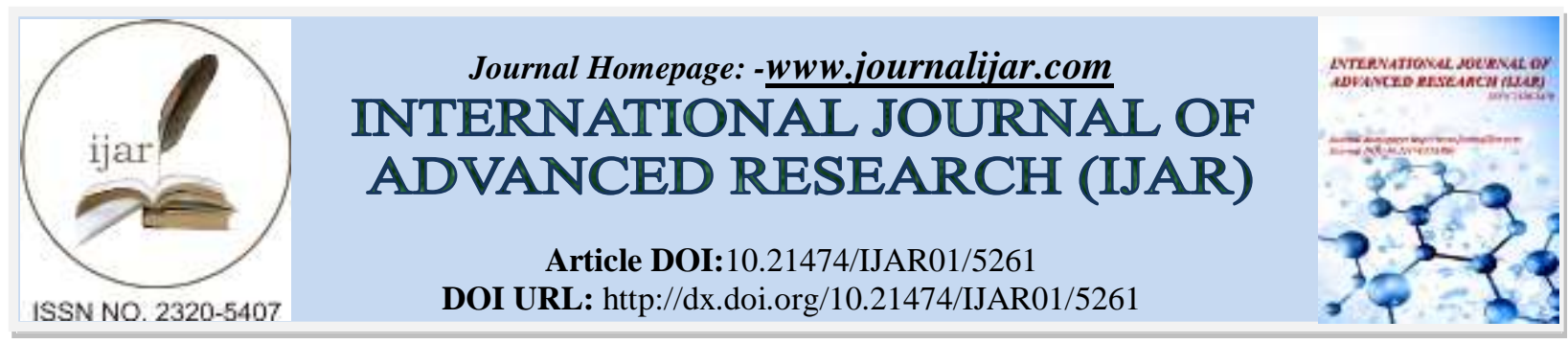

RESEARCH ARTICLE

SUBMISSION OF NEW MANUSCRIPT FOR EVALUATION.

Dr. Mohammad Alajmi.

Ministry of health - kuwait.

\section{Manuscript Info}

Manuscript History

Received: 23 June 2017

Final Accepted: 25 July 2017

Published: August 2017

Keywords:-

Epigenetics; Dna; Amino Acids; Cancer;

Obesity; Ageing.

\section{Abstract}

Epigenetic is referred to as the study of changes which are heritable in gene expression that happen without a DNA sequence change. Effective research has shown that epigenetic mechanisms provide an added layer of transcriptional control which helps regulate the expression of genes. In the normal growth and development of cells, the above mechanisms are said to be very critical components. The abnormalities of epigenetic are said to be causive factors in genetic disorders, cancer and pediatric syndrome. In this paper, we tend to look at the various diseases associated or caused by epigenetic and how they affect the body. The paper will also examine how epigenetic therapy helps combat these diseases.

Epigenetic abnormalities are also said to be a contributing factor to aging and autoimmune diseases. This review will try to examine the major principles of the mechanisms of epigenetic and their different ways of contributing towards the human health together with the consequences of clinical errors in epigenetic. The review will also look at the pathways of epigenetic in the upcoming approaches towards the treatment and also the diagnosis in the clinical spectrum. Diseases that associate with epigenetic come with serious body effects especially those connected with cancer and obesity. This review will give a clear look at the body impact these diseases expose to human beings and how they affect them psychologically and emotionally. The paper will also analyze the research that is being carried out by the medical researchers in order to provide epigenetic treatments to those patients who are affected by epigenetic associated diseases.

Copy Right, IJAR, 2017,. All rights reserved.

\title{
What is Epigenetic?
}

According to Conrad Waddington, he described epigenetic as the biology branch which deals with the study of causal interactions between the genes and genes products which then bring into being the phenotype. This description was stated between 1942.The term epigenetic is dated back in $19^{\text {th }}$ century since it started appearing in the literature since then though the major origin of its concept goes back to Aristotle 384-322 BC. Conrad believed highly in epigenesist which was the individual organic form development from that of the unformed. The argument from against the scientist having developed from fully formed bodies miniscule was due to the controversial view. Currently, the preprogrammed extent versus environmentally shaped still awaits for the consensus universal. The epigenetic field has developed and thus helped bridge the gap that arises between the nature and the nurture. (McVittie, 2006). 
The system of epigenetic should be heritable, reversible and self- perpetuating. However, it is still under debate whether the modification of histone is epigenetic since it is relatively likely that a few of these modifications will be inherited and self perpetuating. Looking further than DNA associated prions, molecules and clearly epigenetic, thus perpetuating themselves by altering the states of folding. Normally, these states act as environmental stress sensors and they drive potentially epigenetic evolution through the promotion of phenotype changes. Rather than a person's sequence of DNA, epigenetic concerns control of genetics by other factors (Allis, 2007). Changes in epigenetic can lead to switching of genes on and off thus help in determining the proteins which will be transcribed. In the twenty first century that we are in epigenetic is defined as the study of changes which are heritable in the function of genome which normally occurs without a DNA sequence change.

Figure 1: It shows the diagram of heritable changes in the genome function that occurs normally without a sequence of a DNA change in the bodies of human beings.

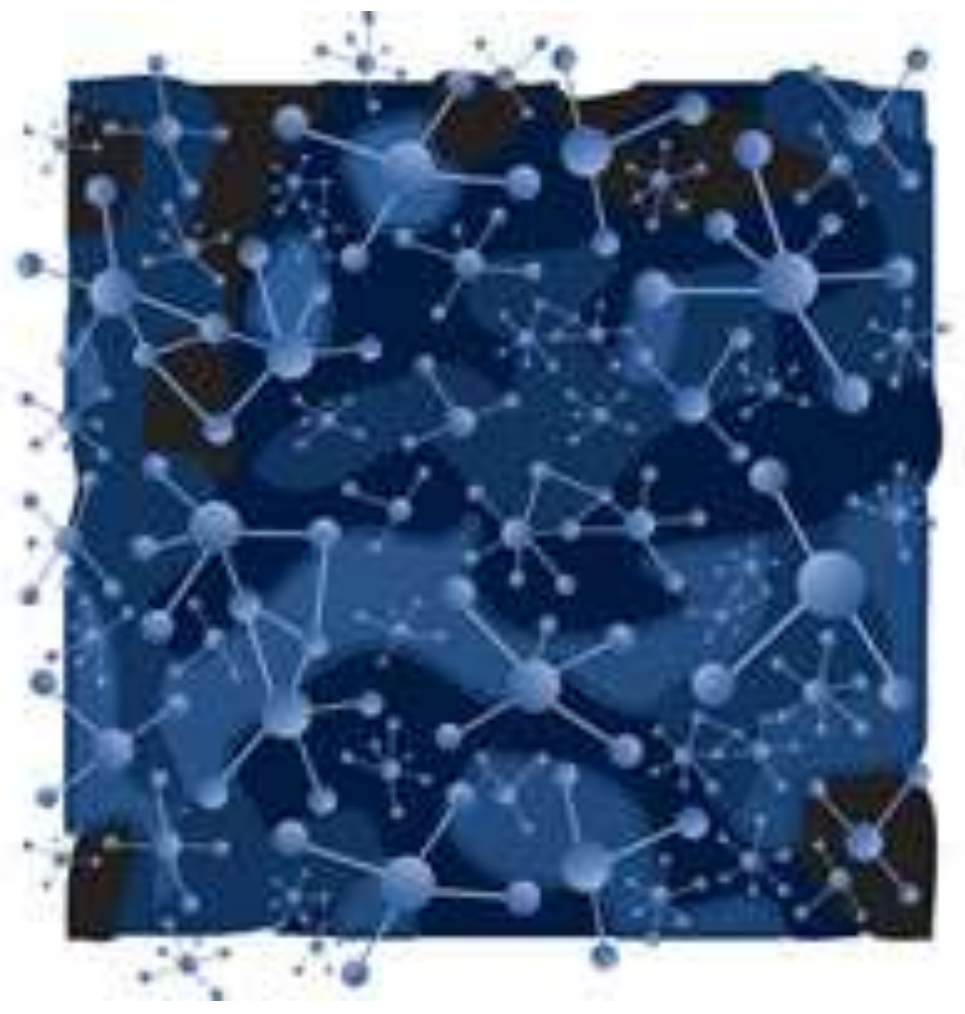

\section{Mechanisms of Epigenetic:-}

Due to intense research by various scientists and medical researchers, there are four types of epigenetic pathways which have been identified. They include:

1. DNA methylation

2. Histone Modification

3. Nucleosome Remodeling

4. Non-coding RNA - mediated pathways

These pathways of epigenetic are known to intertwine with each other in order to enhance the regulation of genes and it is likely possible that other gene will be identified soon apart from the four already identified ones. The most studied and recognize pathways up to date are the histone modification and DNA methylation. Below we briefly describe the two pathways general concepts.

\section{DNA Methylation:-}

Addition of methyl group is done covalently to the cytosine ring fifth carbon in order to enhance formation of 5methyl cytosine. Cytosine is known as one of the five nucleotides found in the RNA and DNA nucleic acids. There are found sites of DNA along the DNA linear chain whereby a cytosine is linked through a phosphate to guanine 
which another known nucleotide. These sites are described as CpG sites and those DNA regions which contain a high density of the $\mathrm{CpG}$ sites are known as $\mathrm{CpG}$ islands. In the $\mathrm{CpG}$ islands is where DNA methylation is known to occur predominately. Cell differentiation and cell functioning is highly contributed to by DNA methylation which is actively involved in their regulation. The functioning of gene can be negated if too little or too little methylation occurs and this can cause unwanted alterations in the cells which can also result to various unwanted diseases.

\section{Histone Modification:-}

Histones are described as the proteins of globular which when they combine with the DNA create the nucleosome a structural unit of chromatin. During the gene transcription phase occurrence, histones influence how loosely or tightly packed the chromatin is. This way the histones are able to influence or trigger whether genes can be transcribed. Enzyme catalyzed addition to or removing of various molecules including acetyl, phosphate, ubiquitin or methyl leads to occurrence of histones modification. If histones are not properly modified then the genes are not expressed properly and clearly as they should normally be or vice versa. Expression of genes in inappropriately levels can cause diseases.

The major histone contains an unstructured $\mathrm{N}$ terminal tail and globular C-terminal domain. A lot of modifications are said to happen in the primarily in the $\mathrm{N}$-terminal tail since there are a variety of modifications taking place in the histone protein. According to the researchers, most of these changes are known to be reversible enzymatically. (Feng, 2006)The biological significance of the modifications is not however, generally understood properly though the modifications are said to influence the transcription, DNA replication, and DNA repair and chromatin condensation.

However, in order to determine whether the histone modifications combinations are essential for predicting the changes in the gene expression, a histone code hypothesis is under test. A good example is lysine acetylation which is highly linked with active DNA which transcriptional and the effects vary by amino acids location, the methyl group number and gene promoter proximity. A review of mechanisms of mammalian epigenetic can help find a comprehensive histone-modifying enzymes list.

Below is a table containing types of histone modification:-

\begin{tabular}{|l|l|}
\hline AMINO ACIDS & MODIFICATION \\
\hline Arginine & methylation \\
\hline Lysine & $\begin{array}{l}\text { Acetylation, } \\
\text { Sumoylation,Ubiquitination and Methylation }\end{array}$ \\
\hline Serine & Phosphorylation \\
\hline Threonine & Phosphorylation \\
\hline
\end{tabular}

\section{Effects of Epigenetic Changes to the Genes:-}

Epigenetic is normally involved in many processes of cellular. The major consideration in this situation is the fact that all our cells have the same DNA but our bodies are made of various types of cells including the liver cells, neurons, inflammatory cells, pancreatic cells and others. The question is how can this be? A simple answer to this is that our tissues, cells and organs are different since they contain various genes sets which are expressed as well as other sets which are inhibited.

One way of turning on genes is through epigenetic silencing and this can highly contribute to various different expressions. Silencing might also give a clear explanation partly as to why genetic twins are non identical phenotypically. Epigenetic is also considered very essential for inactivation of $\mathrm{X}$-chromosomes in female mammals which is very significant so that the females do not contain the number of $\mathrm{X}$-chromosomes genes products twice as the males. Therefore, the implication of turning genes off via epigenetic changes is voluntarily apparent. There are three systems that normally interact with each other inside the cells in order to silence genes. These include histone modification, DNA methylation and RNA-associated silencing

\section{Diseases Associated with Epigenetic:- \\ Epigenetic and Cancer:-}

In 1993 cancer was the first human disease to be associated with epigenetic. The researchers discovered that the tissues which from patients who have colorectal cancer contains less DNA methylation than the normal tissues from the same patients. Since the methylated genes are turned off typically there could be a cause of abnormal high gene 
activation due to loss of DNA methylation. Abnormal high gene activation can happen through altering the chromatin arrangement. Also, too much methylation can undo protective tumor suppressor genes work. In almost every step of tumor development and progression, there is detection of epigenetic changes. Chromosome instability and active oncogenes is highly initiated by too little DNA methylation. It is known that a malignant cell can contain up to twenty to sixty percent less genomic methylation than its normal counterpart.

In other words silencing of tumor suppressor genes can be highly be initiated by too much DNA methylation. Evaluation of epigenic markers is being done by the medical researchers as a means for diagnoising cancer in early stages and prediction of clinical outcome. For effective treatment of cancer, therapeutics based on epigenetic strategies is seriously being considered for the treatment and prevention. (Herceg \& Ushijima, 2010) Changes in epigenetic may be considered as a mechanism of environmental chemical induced cancers though the concept is still under research.

As mentioned previously, occurrence of DNA methylation at $\mathrm{CpG}$ sites and of $\mathrm{CpG}$ cytosine's majority are methylated in mammals. However, near promoter regions there are stretches of DNA that contain higher concentrations of $\mathrm{CpG}$ sites and thus in normal cells they are free of methylation. These CpG islands normally become highly methylated in the cells of cancer and then cause genes which should not be silenced to turn off. This abnormality is then known as the trademark epigenetic change that normally happens in tumors and is known to occur in the early stages during the cancer development. Tumors can also be caused by hypermethylation of $\mathrm{CpG}$ islands through shutting up the genes of tumor-suppressor. In human cancer, these types of changes may be very common than the DNA sequence mutations.

Although changes in epigenetic do not alter the DNA sequence they can otherwise cause mutations. Almost half of the genes that cause either familiar or inherited types of cancer are normally turned off by methylation. Most of these genes are known for suppressing the formation of the tumor and help repair the DNA, including the MLHI cylin-dependent kinase inhibitor 2B (CDKN2B), O6-methylguanine-DNA methyltransferase (MGMT) and RASSF1A.

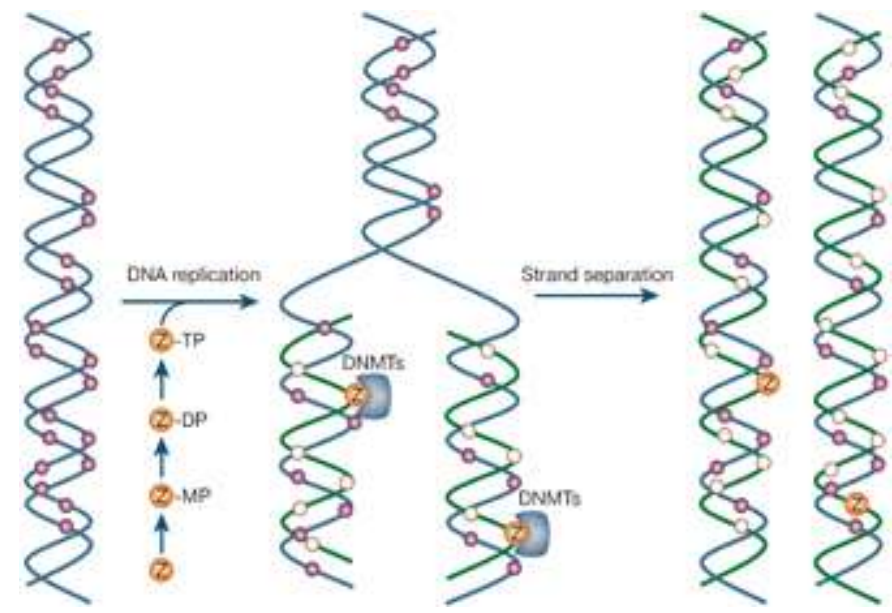

Figure 2:- Mechanism of action of nucleoside analogue inhibitors.

In the figure above, deoxynucleoside analogues including 5-aza-2-deoxycytidine is being converted into triphosphate in the cells of S-phase and then being incorporated into DNA in cytosine place.

Instability of microsatellites can also be caused by hypermethylation which are DNA repeated sequence. Microsatellite are said to be found very common in normal individuals and they normally include dinucleotide CA repeats. Microsatellite can be made unstable and become either shortened or lengthened by too much methylation of DNA repair gene MLHI promoter(Figure 2). The instability of microsatellite has been associated with many cancers including gastric cancers, ovarian cancers, colorectal cancer and endometrial cancers (Jones \& Baylin, 2002).

\section{Cancer Effects to the Body:-}

For the standard development and tissues maintenance in the precise gene expression prototype in mammals epigenetic mechanisms are very important. If the processes of epigenetic are disrupted in any way, this can direct to 
altering of the functions of the gene and the transformation of malignant cellular. Changes in the globe concerning the epigenetic landscape are said to be a major hallmark for cancer. In beginning and development of cancer which is observed as a genetic disease traditionally is now known to involve the abnormalities of epigenetic together with the alterations of genes. Recently, there have been improvement in the fast evolving area of cancer epigenetic and they have really shown wide-ranging reprogramming of all the components of the machinery of epigenetic in cancer consisting DNA methylation, histone modifications, nucleosome positioning and non-codingRNAs, most specifically the expression of microRNA.

However, the reversible character of aberrations of epigenetic has triggered the emergence of epigenetic remedy which is a promising field and has thus made improvement with the current approval of FDA epigenetic drugs used for treatment of cancer. Cancer is considered among the most dangerous terminal diseases in the world currently, and the researchers are doing anything to find its major causes and prevent it.

\section{Epigenetic and Obesity:-}

When the word epigenetic is mentioned, it describes the phenomena of changes which are inherited in the function of gene that independently occur of changes in nucleotide sequence. People believed that the modifications of epigenetic were initially unidirectional but studies recently have described that the epigenome is very dynamic thus changing in regards to the nutrient availability response, aging, physical exercises and other exposures. Different types of cells have their own epigenomes despite the fact that nearly all body cells have similar nuclear genome. The above characteristic is very essential for cell-phenotypes development. DNA methylation occurs to cytosine primarily in CpG-dinucleotides and this happens in different mammalian cells.

There has been an implication of DNA methylation of promoters of gene in transcriptional silencing mostly through the factor of repressed transcription which normally binds to the promoters of gene or through methyl CG binding proteins which recruits histone deacetyltransferases in return and also corepressors. Histone modification by virtue of the capacity of the cell can help control its structure of chromatin and suppress its genes transcription. The nutrition in the early life of an individual characterizes a fascinating example of how augmented epigenetic environmentally events might place an effect on the response of an individual metabolic load and susceptibility of disease in adulthood. However, the understanding of events of epigenetic in the early life manipulates obesity development and its comorbidities remain fairly rudimentary.

Obesity is a very complex disease which has multiple risk factors which are well defined. However, obesity susceptibility and its sequelae within the environment of obesogenic diverge very much from one individual to the other thus suggesting the gene $\mathrm{x}$ role and its interactions in the environment in the etiology of disorder. Putative mechanism is provided by the regulation of epigenetic in the human body whereby specific exposures of the environment trigger the obesity risk and other diseases that affect human beings and it is a mechanism which can possibly underlie the environment or treatment of gene $\mathrm{x}$ interactions which are normally observed the studies and clinical trials of epidemiology. BMC Medicine published a study regarding the examination of DNA methylation in peripheral blood leukocytes of lean and adolescents who are obese thus putting into comparison the patterns of methylation between two or more groups. There was identification of two genes which were said to be methylated differently and both had effective roles in the function of immune.

\section{Effects of Obesity to the Body:-}

Obesity can bring a lot of harm to the human body. Some of the harm that obesity can be related to include:

1. Changes in the normal functions of the body: The body changes vary from one individual to another depending also with the degree of obesity. People with obesity are faced with respiratory problems which a very common issue. Another major body function that is recognized include abnormal clotting of the blood, the person affected becoming lethargic, heart enlargement, congestive heart failure and lower tolerance in exercises.

2. Increased Risk of various Diseases: Obesity is highly associated with various chronic diseases which are degenerative. Some of these diseases include hypertension, coronary diseases, arthritis which is caused by extra load on the joints because of overweight and diabetes.

3. Detrimental impacts on Diseases which are established: Certain medical conditions and diseases can highly rise due to obesity and reduction of weight is normally prescribed as a part of treatment for certain diseases. Some conditions that led to prescription of weight loss as a priority include diabetes, congestive heart disease, heart attack, hypertension and orthopedic problems. 
4. Emotional Effect: Obesity can affect an individual psychologically and emotionally thus bringing problems which might be as a result of the disease. Obesity carries a social stigma in the today's society and this enhances to psychological reactions for those people affected by obesity. This is mostly seen in the media which glamorizes those people who only have slim figures and lean bodies.

\section{Epigenetic and Aging:-}

The steady state of histone acetylation level is highly determined by the histone transferases (HATs), histone deacetylases (HDACs) and acetyl groups respectively. Replicative lifespan is shortened by inactivation of histone deacetylase, Sir2 while activation of Sir2 lengthens the lifespan. Certain anti-aging effects in Sir2 yeast are partly because of Sir2 translocation which contains complex proteins from telomeres to repeats of DNA ribosomal. The repeats are subjected to formation of extra chromosomal ribosomal DNA cycles after recombination which curtail the lifespan of yeast. Prevention of recombination and ERCs formation is done at the repeat of ribosomal DNA and consequent heterochromatinization thus extending the lifespan (Tollefsbol, 2010).

Yeast aging is linked with redistribution of nuclear of a histone enzyme modifying in sum, Sir2 and heterochromatin binding proteins. Organismal aging is counteracted by the epigenetic redistribution (Sedivy, Banumathy, \& Adams, 2008)Aging is a painful reality to many since it comes with other shortcomings mostly related with memory loss, loss of eyesight, wrinkles or physical change and so many other effects to the body. Complex events of developmental series mark the individual's normal lifespan. Another major developmental event that marks the human lifespan includes a gradual decline in viability and relative stability during adulthood.

\section{Epigenetic and the Environment:-}

There are certain dietary and environmental factors which are linked to abnormal changes in epigenetic pathways in epidemiological studies and experimental studies. It is difficult sometimes to create a clear-cut-causal relationship between the disease and the dietary factor and this is because the epigenetic changes are cumulative and subtle thus manifesting overtime. Some of the examples of environmental factors that are related to epigenetic pathway changes include:

1. Heavy metals can disrupt DNA methylation

2. Vinclozolin. It alters DNA methylation in exposed laboratory animals.

3. Deficiencies in folate and methionine

4. Cigarette smoke. It stimulates the demethylation of genes of metastatic in lung cancer cells.

\section{Combating Diseases with Epigenetic Therapy:-}

Due to so many evolving diseases worldwide today including cancer which is involved in epigenetic change, it has become so reasonable to try and counteract these modifications by finding epigenetic treatments. These changes have been regarded a very ideal target since they are known to be reversible in nature compared to DNA sequence mutations. (Gasser, 2010)This major treatment tends to aim at altering the histone acetylation or DNA methylation. Genes that have been silenced can be reactivated by the inhibitors of DNA methylation. The two types of known drugs include 5-aza-2 and 5-aza-2. The medications work through acting like the nucleotide cytosine and also incorporate themselves in the DNA as it replicates. The drugs then block the DNMT enzymes and prevent them from acting thus inhibiting the DNA methylation and this is after they are incorporated.

Drugs which are manufactured for the modification of histone are known as histone deacetylase (HDAC) inhibitors. These are known as enzymes that remove the group of acetyl from DNA which then condenses the chromatin and leads to stopping of transcription. The gene expression is then turned on by blocking the process of HDAC inhibitors. However, the commonest known HDAC inhibitors are known as SAHA, valproic acid, phenylbutyric acid and depsipeptide (Simmons, 2008).

It is necessary to use epigenetic therapy since the processes of epigenetic and its changes are known to be very widespread. In order for the epigenetic treatments to be successful, they must be very selective towards the irregular cells otherwise gene transcription activation could make them cancerous in normal cells and therefore the treatment can cause the same disorders which they are trying to neutralize (Herceg \& Ushijima, Epigenetics and Cancer, 2010). Researchers are therefore looking for ways and means of targeting the abnormal cells specifically despite the possible problems they are facing and this is being done in a way that they will put little damage to the normal cells and therefore the process of epigenetic therapy is becoming more promising more and more each day. 


\section{References:-}

1. Allis, C. D. (2007). Epigenetics. New York: CSHL Press.

2. Feng, Y.-W. (2006). Ethanol-induced p53 and histone modifications. In Y.-W. Feng, Ethanol-induced p53 and histone modifications (p. 28). California: ProQuest Information and Learning Company.

3. Gasser, S. (2010). Epigenetics and Disease: Pharmaceutical Opportunities. In S. Gasser, Epigenetics and Disease: Pharmaceutical Opportunities (p. 270). London: Springer Publishers.

4. Herceg, Z., \& Ushijima, T. (2010). Epigenetics and Cance. San Diego: Elsevier, Inc.

5. Herceg, Z., \& Ushijima, T. (2010). Epigenetics and Cancer. In Z. Herceg, \& T. Ushijima, Epigenetics and Cancer (p. 327). Academic Press.

6. McVittie, B. (2006, June). What is Epigenetics? . Retrieved March 17, 2012, from Epigenetics: http://epigenome.eu/en/1,1,0

7. Rodenhiser, D., \& Mann, M. (2006). Epigenetics and human disease: translating basic biology into clinical applications. Canadian Medical Association Journal, vol. 174 no. 3.

8. Sedivy, J. M., Banumathy, G., \& Adams, P. D. (2008). Aging by epigenetics - a consequence of chromatin damage? NIHPA, 1909-1917.

9. Simmons, D. (2008). Epigenetic Influences and Disease. Retrieved March 17, 2012, from Nature Education: http://www.nature.com/scitable/topicpage/epigenetic-influences-and-disease-895

10. Tollefsbol, T. O. (2010). Epigenetics of Aging. London: Springer Publishers. 